\title{
ON THE NETTO INVERSION NUMBER OF A SEQUENCE
}

\author{
DOMINIQUE FOATA
}

1. Introduction. Let $g=\left(x_{1}, x_{2}, \cdots, x_{n}\right)$ be an arbitrary sequence of real numbers and $\mathfrak{e}$ the set of all sequences that can be formed from $g$ by permutations. If $f=\left(x_{i_{1}}, x_{i_{2}}, \cdots, x_{i_{n}}\right)$ is in $\mathcal{C}$, the inversion number $S(f)$ of $f$ is defined as the number of couples $(j, k)$ such that $1 \leqq j<k \leqq n$ and $x_{i_{j}}>x_{i_{k}}$ and the index $T(f)$ of $f$ as the sum of all integers $j$ such that $1 \leqq j \leqq n-1$ and $x_{i_{j}}>x_{i_{j+1}}$.

The function $S$ seems to have been introduced by Netto [6] and rediscovered many times in statistics in the theory of rank tests. It also appears in the so-called two-sample problem under the name of Wilcoxon-Mann-Whitney statistic (see e.g. [1]).

MacMahon ([3], [4]) introduced the function $T$ in the study of ordered partitions. Let $q$ be a real or complex variable and $S=\sum\left\{q^{S(f)}: f \in \mathcal{C}\right\}$ (resp. $\boldsymbol{T}=\sum\left\{q^{T(f)}: f \in \mathcal{C}\right\}$ ) be the generating function of $S$ (resp. $T$ ). He then obtained [5] the surprising result that $S$ and $T$ have the same expression. Hence the fact that

(1.1) for any nonnegative integer $m$ there are in $\mathcal{C}$ as many sequences $f$ such that $S(f)=m$ as sequences $f^{\prime}$ such that $T\left(f^{\prime}\right)=m$.

It seems that no explicit one-to-one correspondence has been so far given between the set of sequences for which $T$ is equal to $m$ and the set of sequences for which $S$ is equal to $m$. The purpose of the present paper is to give the construction of such a correspondence. This construction, without fully explaining the above result (1.1), allows us to introduce a new class of rearrangements of sequences and apply the same noncommutative algebraic methods as in [2].

In what follows, it will be more convenient to identify a sequence $f=\left(x_{i_{1}}, x_{i_{2}}, \cdots, x_{i_{n}}\right)$ of $\mathfrak{C}$ with the associative monomial or word $x_{i_{1}} x_{i_{2}} \cdots x_{i_{n}}$ of the free monoid $X^{*}$ generated by $X=R$, to extend the definition of $S$ and $T$ to all of $X^{*}$ and to construct a permutation $\Phi$ of $X^{*}$ satisfying

$$
S(\Phi(f))=T(f) \quad \text { for all } f \in X^{*}
$$

and such that if $f=x_{i_{1}} x_{i_{2}} \cdots x_{i_{n}}$, then $\Phi(f)=x_{v_{1}} x_{v_{2}} \cdots x_{v_{n}}$ where $\left(x_{v_{1}}, x_{v_{2}}, \cdots, x_{v_{n}}\right)$ is a permutation of $\left(x_{i_{1}}, x_{i_{2}}, \cdots, x_{i_{n}}\right)$.

The definitions and notations being given in $\$ 2$, a set of permutations $\left(\gamma_{x}\right)_{x \in X}$ of $X^{*}$ is introduced ( $\left.\$ 3\right)$ and the permutation $\Phi$ is defined in $\$ 4$ by induction on the length of the words of $X^{*}$, i.e. for all $x \in X$ and $f \in X^{*}$, we set

Received by the editors April 26, 1966. 


$$
\Phi(x)=x \quad \text { and } \quad \Phi(f x)=\gamma_{x}(\Phi(f)) x .
$$

2. Notations and definitions. In what follows, $X^{*}$ is the free monoid generated by a totally ordered set $X$. Each element $f$ of $X^{*}$ can be written as a word $f=x_{1} x_{2} \cdots x_{n}$ where $x_{1}, x_{2}, \cdots, x_{n}$ belong to $X$ and are the $n$ letters of the word and where $n$ is a nonnegative integer, by definition equal to the length of $f$, denoted by $\lambda f$. The word of length 0 is the empty word denoted by $I$. The words of length $n(n \geqq 0)$ constitute a subset of $X^{*}$ denoted by $X_{n}$ and $X_{1}$ is identified with $X$. If $f$ is the product of $s(s \geqq 2)$ words $f_{1}, f_{2}, \cdots, f_{s}$ of $X^{*}$, we write $f=f_{1} f_{2} \cdots f_{s}$ and as in [7] we say that $f_{1} f_{2} \cdots f_{s}$ is a factorization of $f$. The word $f$ is also a factorization of itself.

Moreover if $Y$ and $Z$ are subsets of $X^{*}$, we designate by $Y^{*}$ the submonoid generated by $Y$ and by $Y Z$ the subset of the words $f=f^{\prime} f^{\prime \prime}$ with $f^{\prime} \in Y$ and $f^{\prime \prime} \in Z$. Thus $X X^{*}\left(=X^{*} X\right)$ is the subset of words of positive length. Now since $X$ is totally ordered, each $x \in X$ determines a partition of $X$ in two subsets $L_{x}$ and $R_{x}$. The set $\left.L_{x}=\right] \leftarrow$, $x]$ (resp. $\left.R_{x}=\right] x, \rightarrow[$ ) is formed with all $y \in X$ such that $y \leqq x$ (resp. $y>x$ ). Then for each $x \in X$ and $f=x_{1} x_{2} \cdots x_{n} \in X^{*}$, we denote by $l_{x} f$ (resp. $r_{x} f$ ) the number of subscripts $j$ for which $1 \leqq j \leqq n$ and $x_{j} \leqq x$ (resp. $x<x_{j}$ ). Note that we always have $l_{x} f+r_{x} f=\lambda f$. If $l_{x} f=l_{x} f^{\prime}$ for all $x \in X$ or if $f^{\prime}$ is a rearrangement of the letters of $f$, we set $\alpha(f)=\alpha\left(f^{\prime}\right)$.

Finally for $f=x_{1} x_{2} \cdots x_{n} \in X^{*}$, we set

$S(f)=$ number of couples $(j, k)$ such that $1 \leqq j<k \leqq n$ and $x_{j}>x_{k}$. $T(f)=$ sum of all integers $j$ such that $1 \leqq j \leqq n-1$ and $x_{j}>x_{j+1}$.

$$
\begin{aligned}
f^{\pi} & =f \quad \text { if } n=0 \text { or } 1, \\
& =x_{n} x_{1} x_{2} \cdots x_{n-1} \text { if } n>1 .
\end{aligned}
$$

3. The set of permutations $\left(\gamma_{x}\right)_{x \in X}$. First, it is obvious that for every $x \in X$,

$$
\left\{X^{*} L_{x}, X^{*} R_{x}\right\} \quad \text { and } \quad\left\{L_{x} X^{*}, R_{x} X^{*}\right\}
$$

are two partitions of $X^{*} X\left(=X X^{*}\right)$. Moreover, let $f=x_{1} x_{2} \cdots x_{n}$ be a word of $X^{*} L_{x}$ (resp. $X^{*} R_{x}, L_{x} X^{*}, R_{x} X^{*}$ ) and denote by $\left(r_{1}, r_{2}, \cdots, r_{s}\right)$ the increasing sequence of integers $j(1 \leqq j \leqq n)$ such that $x_{j} \in L_{x}$ (resp. $R_{x}, L_{x}, R_{x}$ ). This sequence is not empty. Put $r_{0}=0, r_{s+1}=n+1$ and for $p=1,2, \cdots, s$

$$
f_{p}=x_{r_{p-1}+1} x_{r_{p-1+2}} \cdots x_{r_{p}} \text { if } f \in X^{*} L_{x} \text { or } f \in X^{*} R_{x}
$$

and

$$
f_{p}=x_{r_{p}} x_{r_{p}+1} \cdots x_{r_{p+1}-1} \text { if } f \in L_{x} X^{*} \text { or } f \in R_{x} X^{*} .
$$


Clearly, $f_{1} f_{2} \cdots f_{s}$ is the unique factorization of $f$ where each $f_{p} \in R_{x}^{*} L_{x}$ (resp. $L_{x}^{*} R_{x}, L_{x} R_{x}^{*}, R_{x} L_{x}^{*}$ ).

This factorization will now be used for establishing a one-to-one correspondence between $X^{*} L_{x}$ and $L_{x} X^{*}$ on one hand, and $X^{*} R_{x}$ and $R_{x} X^{*}$ on the other hand and so defining a permutation $\gamma_{x}$ of $X^{*}$. First we set $\gamma_{x}(I)=I$, then if $f_{1} f_{2} \cdots f_{s}$ is the factorization of a word $f \in X^{*} L_{x}$ (resp. $X^{*} R_{x}$ ) into words of $R_{x}^{*} L_{x}$ (resp. $L_{x}^{*} R_{x}$ ), we set

$$
\gamma_{x}(f)=f_{1}^{\pi} f_{2}^{\pi} \cdots f_{s}^{\pi} .
$$

We have $f_{p}^{\pi} \in L_{x} R_{x}^{*}$ (resp. $R_{x} L_{x}^{*}$ ) for $p=1, \cdots, s$; hence from above $f_{1} f_{2} \cdots f_{p}$ is the factorization of a unique word $\gamma_{x}(f) \in L_{x} X^{*}$ (resp. $R_{x} X^{*}$ ) into words of $L_{x} R_{x}^{*}$ (resp. $R_{x} L_{x}^{*}$ ). Finally, as $h \rightarrow h^{*}$ maps in a one-to-one manner $L_{x}^{*} R_{x}$ onto $R_{x} L_{x}^{*}$ and $R_{x}^{*} L_{x}$ on to $L_{x} R_{x}^{*}$, $\gamma_{x}$ is a permutation of $X^{*}$ and besides, for every $f \in X^{*}, \gamma_{x}(f)$ is a rearrangement of the letters of $f$, i.e.

$$
\alpha\left(\gamma_{x}(f)\right)=\alpha(f) .
$$

Before introducing the permutation $\Phi$, we give in the following lemma some properties of the functions $S$ and $T$.

(3.3) Lemma. For each $f \in X^{*}$ and $x \in X$,

$$
\begin{aligned}
S(f x) & =S(f)+r_{x} f, & & \\
S\left(\gamma_{x}(f)\right) & =S(f)-r_{x} f & & \text { if } f \in X^{*} L_{x}, \\
S\left(\gamma_{x}(f)\right) & =S(f)+l_{x} f & & \text { if } f \in X^{*} R_{x}, \\
T(f x) & =T(f) & & \text { if } f \in X^{*} L_{x}, \\
T(f x) & =T(f)+\lambda f & & \text { if } f \in X^{*} R_{x} .
\end{aligned}
$$

Proof. Let $f=x_{1} x_{2} \cdots x_{n} \in X^{*}$.

First, (3.4) holds for the inversion number of $f x$ is equal to the inversion number of $f$, plus the number of subscripts $j(1 \leqq j \leqq n)$ such that $x_{j}>x$, i.e. $r_{x} f$.

Now if $f \in R_{x}^{*} L_{x}$, we can write $f=f^{\prime} x_{n}\left(f^{\prime} \in R_{x}^{*}, x_{n} \leqq x\right)$; thence

$$
r_{x} f=r_{x_{n}} f=r_{x_{n}} f^{\prime}=\lambda f^{\prime} .
$$

But $\gamma_{x}(f)=f^{x}=x_{n} f^{\prime}$. Thus $S\left(\gamma_{x}(f)\right)$ is equal to the inversion number of $f^{\prime}$ plus if $n>1$, the number of subscripts $j(1 \leqq j \leqq n-1)$ such that $x_{n}>x_{j}$, which is 0 since $f^{\prime} \in R_{x}^{*}$, i.e.

$$
\begin{array}{rlrl}
S\left(\gamma_{x}(f)\right) & =S\left(x_{n} f^{\prime}\right)=S\left(f^{\prime}\right) & \\
& =S\left(f^{\prime} x_{n}\right)-r_{x_{n}} f^{\prime} & & \text { from (3.4) } \\
& =S(f)-r_{x} f & & \text { from (3.9); }
\end{array}
$$


(3.5) is then true for the words $f \in R_{x}^{*} L_{x}$. Finally, if $f \in X^{*} L_{x}$, let $f_{1} f_{2} \cdots f_{s}$ be its factorization into words of $R_{x}^{*} L_{x}$. By applying $\gamma_{x}$ to $f$, we obtain $\gamma_{x}(f)=f_{1}^{\pi} f_{2}^{\pi} \cdots f_{s}^{\pi}$ and clearly the inversion number of $f$ is decreased by $r_{x} f_{1}+r_{x} f_{2}+\cdots+r_{x} f_{s}$, i.e. $r_{x} f$.

(3.6) has an analogous proof. We simply notice that applying $\gamma_{x}$ to a word $f$ of $L_{x}^{*} R_{x}$, increases the inversion number by $\lambda f-1$, or $l_{x} f$.

When $f \in X^{*} L_{x}$, the last letter $x_{n}$ of $f$ is less than or equal to $x$ and the indices of $f$ and $f x$ are the same. Hence (3.7) holds.

On the contrary, if $f \in X^{*} R_{x}$, then $x_{n}>x$ and we get

$$
T(f x)=T(f)+\lambda f .
$$

That is (3.8), which completes the proof of the lemma.

4. The combinatorial theorem. By induction on the length of words $f \in X^{*}$, we then define $\Phi$ in the following way:

$$
\Phi(f)=f \text { if } \lambda f \leqq 1
$$

and

$$
\Phi(f x)=\gamma_{x}(\Phi(f)) x \text { for all } x \in X .
$$

We then have

(4.3) Theorem. The map $\Phi: X^{*} \rightarrow X^{*}$ is a permutation; and we have

$$
\begin{gathered}
\alpha(\Phi(f))=\alpha(f) \\
S(\Phi(f))=T(f) \text { identically. }
\end{gathered}
$$

Proof. It is sufficient to verify that for all $n \geqq 0$ the restriction $\Phi_{n}$ of $\Phi$ to $X_{n}$ is a permutation of $X_{n}$ satisfying (4.5) and (4.6). This is obvious for $n \leqq 1$ since by definition $\Phi_{0}$ (or $\Phi_{1}$ ) is the identity map. On the other hand from the definition of $\Phi$ we have for $n>0$,

$$
\Phi_{n+1}(f x)=\gamma_{x}\left(\Phi_{n}(f)\right) x,
$$

valid for all $f \in X_{n}$ and $x \in X$.

So assume that $\Phi_{n}$ is a permutation of $X_{n}$ satisfying $\alpha\left(\Phi_{n}(f)\right)=\alpha(f)$ and $S\left(\Phi_{n}(f)\right)=T(f)$ identically. Then $\gamma_{x} \circ \Phi_{n}$ is also a permutation of $X_{n}$ and satisfies $\alpha\left(\gamma_{x}\left(\Phi_{n}(f)\right)\right)=\alpha(f)$ identically according to (4.7).

Hence, $f x \rightarrow \Phi_{n+1}(f x)=\gamma_{x}\left(\Phi_{n}(f)\right) x$ is a permutation of the subset of the words of $X_{n}$ ending by $x$ and also $\alpha\left(\Phi_{n+1}(f x)\right)=\alpha(f x)$.

Since $X_{n+1}=\cup_{x \in X} X_{n}\{x\}$, it then follows that $\Phi_{n+1}$ is a permutation of $X_{n+1}$ satisfying

$$
\alpha\left(\Phi_{n+1}(f)\right)=\alpha(f) \text { identically. }
$$


Property (4.6) is then a consequence of the lemma. For

$$
\begin{aligned}
S\left(\Phi_{n+1}(f x)\right) & =S\left(\gamma_{x}\left(\Phi_{n}(f)\right) x\right) \\
& =S\left(\gamma_{x}\left(\Phi_{n}(f)\right)\right)+r_{x} \gamma_{x}\left(\Phi_{n}(f)\right) \quad \text { (according to (3.4)) } \\
& =S\left(\gamma_{x}\left(\Phi_{n}(f)\right)\right)+r_{x} f
\end{aligned}
$$

since $\gamma_{x}\left(\Phi_{n}(f)\right)$ is only a rearrangement of the letters of $f$.

Two cases are to be considered.

(i) $f \in X^{*} L_{x}$. Then

$$
\begin{aligned}
S\left(\gamma_{x}\left(\Phi_{n}(f)\right)\right) & =S\left(\Phi_{n}(f)\right)-r_{x} \Phi_{n}(f) \quad \text { (according to (3.5)) } \\
& =S\left(\Phi_{n}(f)\right)-r_{x} f .
\end{aligned}
$$

Hence,

$$
\begin{aligned}
S\left(\Phi_{n+1}(f x)\right) & =S\left(\Phi_{n}(f)\right) \\
& =T(f) \quad \text { (by induction) } \\
& =T(f x) \quad \text { (according to (3.7)). }
\end{aligned}
$$

(ii) $f \in X^{*} R_{x}$. Then

$$
\begin{aligned}
S\left(\gamma\left({ }_{x} \Phi_{n}(f)\right)\right) & =S\left(\Phi_{n}(f)\right)+l_{x} \Phi_{n}(f) \quad \text { (according to (3.6)) } \\
& =S\left(\Phi_{n}(f)\right)+l_{x} f .
\end{aligned}
$$

Hence,

$$
\begin{aligned}
S\left(\Phi_{n+1}(f x)\right) & =S\left(\Phi_{n}(f)\right)+l_{x} f+r_{x} f \\
& =T(f)+\lambda f \quad \text { (by induction) } \\
& =T(f x) \quad \text { (according to (3.8)). }
\end{aligned}
$$

This establishes the theorem.

\section{REFERENCES}

1. F. N. David and D. E. Barton, Combinatorial chance, Griffin, London, 1962.

2. D. Foata, Étude algébrique de certains problèmes d'analyse combinatoire et du calcul des probabilités, Publ. Inst. Statist. Univ. Paris 14 (1965), 81-241.

3. P. A. MacMahon, Combinatory analysis, Vol. 1, Cambridge Univ. Press, Cambridge, 1915.

4. - The indices of permutations and the derivation therefrom of functions of a single variable associated with the permutations of any assemblage of objects, Amer. J. Math 35 (1913), 281-322.

5. - Two applications of general theorems in combinatory analysis, Proc. London Math. Soc. 15 (1916), 314-321.

6. E. Netto, Lehrbuch der Combinatorik, Chelsea, New York, 1901.

7. M. P. Schützenberger, On a factorisation of free monoids, Proc. Amer. Math. Soc. 16 (1965), 21-24.

Institut de Recherche Mathématique Avancée, Université de Strasbourg 